\title{
PRESENTATIONS OF SOME FINITE SIMPLE GROUPS
}

\author{
DRAGOMIR Ž. ĐOKOVIĆ
}

(Received 18 July 1986)

Communicated by H. Lausch

\begin{abstract}
We give new presentations of the five Mathieu groups, the simple groups $J_{1}, J_{2}, H S, M c L$, $\mathrm{Co}_{3}$, and some other simple and related groups. All generators in these presentations are involutions. Our presentations are simpler than the known presentations of this type for the groups mentioned above.
\end{abstract}

1980 Mathematics subject classification (Amer. Math. Soc.): 20 D 08, 20 F 05.

\section{Introduction}

In this paper we give a number of presentations of sporadic simple groups of low order (the Mathieu groups, $J_{1}, J_{2}, \mathrm{HS}, \mathrm{McL}, \mathrm{Co}_{3}$ ) and some other simple and related groups. For some of them we give several presentations, for instance Section 1 contains three different presentations of the Mathieu group $M_{11}$. All generators in our presentations are involutions and we shall refer to such presentations as Coxeter type presentations. For a large number of finite simple and related groups (covering groups or automorphism groups of simple groups, etc.) such presentations are given in the recent Atlas of Finite Groups by J. H. Conway et al. [4]. Many of the presentations in this Atlas are due to L. Soicher and appear in his thesis [5], see also his preprint [7].

Our goal was to obtain simpler presentations than those in the Atlas and also to find Coxeter type presentations for some simple groups for which no such

(C) 1988 Australian Mathematical Society $0263-6115 / 88 \$ A 2.00+0.00$ 
presentation is given in the Atlas. In order to attach an objective meaning to the word "simpler" we introduce the notion of the length of a presentation. Then "simpler" means just "shorter". Let $G=\langle S: R\rangle$ be a presentation of $G$. Each relator $r \in R$ is an element of the free groups on $S$. If $l(r)$ is the length of $r$ as an element of that free group, then the length of the presentation $\langle S: R\rangle$ is the number

$$
|R|+\sum_{r \in R} l(r)
$$

(If we view our presentation as a character string which is fed into a computer then we need separators to separate the defining relators from each other. This explains the presence of the term $|R|$ in the above expression.)

We mention that the problem of finding a shortest presentation for a given finite group $G$ is quite open. We do not know the answer even in the case when $G$ is a cyclic group of prime order.

One possible use of presentations of groups in computer systems for group theory is the following (suggested by L. Soicher). Assume that $G$ has a faithful transitive permutation representation of degree $d$ (not too big). Then a usual way to store $G$ in a computer file is to store the generators of $G$ as permutations on $\{1,2, \ldots, d\}$. A better way is to just store a presentation of $G$, say $\langle S: R\rangle$ and a set of generators of a one point stabilizer in that permutation representation. Then one can recover the permutation representation by a coset enumeration procedure, for example, the standard function "cosact image" in CAYLEY. (For a description of CAYLEY see [1].) The advantage of the latter method is that the file containing the presentation and the necessary commands to generate the premutation representation is much shorter than the file containing the list of generators of $G$ as permutations. Moreover the presentation file is more reliable since it is easier to understand and to verify its correctness. (We have made a comparison in the case of the Conway group $\mathrm{Co}_{3}$ for which $d=276$. In that case our presentation file consists of 10 lines while the permutation file cannot fit on one screen.)

Our presentations, $G=\langle S: R\rangle$, have the following form. We first give a Coxeter diagram for $S=\left\{x_{1}, \ldots, x_{n}\right\}$. More precisely, each (involutory) generator is represented by a node in this diagram; two nodes $x_{i}$ and $x_{j}$ are joined by an edge if and only if the order $m_{i j}$ of the product $x_{i} x_{j}$ is $\geq 3$, and in case $m_{i j}>3$ the label $m_{i j}$ is attached to this edge.

Next we list the set of generators $S$, and the defining relators $R$ of $G$. In all cases we have tried to reduce $R$ to be a minimal set of defining relators but we do not make any such claim. In fact, we believe that in many cases $R$ is not minimal. As an example we mention the case of the presentation (13.1) of $\mathrm{Co}_{3}$. Originally that presentation included the redundant relator $a(c d e f)^{4}$ and then the enumeration of 170,775 cosets of $\langle a, b, c, e, f, g\rangle \cong 2 \cdot S_{6}(2)$ in $\mathrm{Co}_{3}$ 
took less than $9 \mathrm{~h}$. of CPU-time. When we deleted this relator then the same enumeration took more than $20 \mathrm{~h}$. of $\mathrm{CPU}$-time. (Moreover we had to make some improvements in our program in order to be able to complete this last enumeration.) All computations were carried out on a VAX 11/785 computer running VMS V4.4.

Finally for some subsets $T$ of $S$ we describe the subgroup of $G$ generated by $T$ and we list some consequences of $R$.

In the case of Mathieu groups or their covering groups we also specify a homomorphism of $G$ onto the corresponding Mathieu group, the latter group we consider as being given by its standard definition via the MOG or MINIMOG arrays, see [2], [3] or [5]. For the convenience of the reader we give in Table 1 the labellings for these arrays which exhibit the maximal subgroups $L_{2}(23)$ and $L_{2}(11)$ of $M_{24}$ and $M_{12}$, respectively, see [3].

TABLE 1

MOG

MINIMOG

$\begin{array}{rrrrrrrrrr}0 & \infty & 1 & 11 & 2 & 22 & & & & \\ 19 & 3 & 20 & 4 & 10 & 18 & 0 & 3 & \infty & 2 \\ 15 & 6 & 14 & 16 & 17 & 8 & 5 & 9 & 8 & 10 \\ 5 & 9 & 21 & 13 & 7 & 12 & 4 & 1 & 6 & 7\end{array}$

All presentations given below were obtained by using CAYLEY and the author's version of the Todd-Coxeter coset enumeration procedure. One can verify the correctness of the listed presentations by using CAYLEY, the Todd-Coxeter procedure, and the recognition theorems for the relevant simple groups. In the last section of the paper we give details of such verifications for the presentations (7.1), (8.1), and (10.1) of $M_{24}, J_{1}$, and $H S$, respectively.

TABLE 2

Lengths

\begin{tabular}{lrrr} 
Group & New & Atlas & Pres \\
\hline$M_{11}$ & 64 & 68 & $(1.1)$ \\
$M_{12}$ & 76 & 125 & $(2.1)$ \\
$M_{21}$ & 116 & - & $(3.2)$ \\
$2^{2} \cdot M_{21}$ & 68 & - & $(3.1)$ \\
$A_{8}$ & 103 & - & $(4.1)$ \\
$M_{22}$ & 108 & 173 & $(5.1)$ \\
$2 \cdot M_{22}$ & 103 & - & $(5.2)$ \\
$6 \cdot M_{22}$ & 91 & - & $(5.4)$ \\
$M_{23}$ & 128 & 146 & $(6.1)$ \\
$M_{24}$ & 127 & 162 & $(7.1)$ \\
$J_{1}$ & 88 & 96 & $(8.1)$
\end{tabular}

Lengths

\begin{tabular}{lrrr} 
Group & New & Atlas & Pres. \\
\hline$J_{2}$ & 95 & 134 & $(9.1)$ \\
$H S$ & 119 & 126 & $(10.1)$ \\
$M c L$ & 119 & 160 & $(11.1)$ \\
$S_{6}(2)$ & 121 & - & $(12.2)$ \\
$2 \cdot S_{6}(2)$ & 142 & - & $(12.4)$ \\
$2 \times S_{6}(2)$ & 92 & 138 & $(12.1)$ \\
$2 \times 2 \cdot S_{6}(2)$ & 112 & - & $(12.6)$ \\
$C o_{3}$ & 174 & 178 & $(13.1)$ \\
$U_{3}(5)$ & 106 & - & $(14.1)$ \\
$U_{3}(5): 2$ & 102 & - & $(14.2)$ \\
$4 \cdot U_{4}(3): 2^{2}$ & 135 & - & $(14.3)$
\end{tabular}


In Table 2 we list all groups $G$ for which we provide new presentations. Following the name of the group we give, respectively, the lengths of our shortest presentation of $G$ and the Atlas presentation of $G$ (if available as Coxeter type presentation). In fairness one should remark that the Atlas presentations were not necessarily made with the aim of being as short as possible. The column labelled "Pres." gives the subsection of this paper containing our shortest presentation of $G$.

For describing the group structures we follow the notation given in the Atlas, p. xx. A brief summary of the notations is given below. We wish to acknowledge the generous support for this work which was provided throught the WATDEC Research Project. Without many days of free CPU-time, provided by this project, the presentations listed in this paper could not have been discovered. This work was also supported by the NSERC Grant A-5285. We are grateful to L. Soicher for sending us a copy of his thesis and for several helpful discussions.

\section{Notations}

$n \quad$ denotes a cyclic group of order $n$.

$G^{k} \quad$ denotes direct product of $k$ copies of $G$.

$A \times B$ is the direct product of $A$ and $B$.

$n^{k} \quad$ is a special case of $G^{k}$, i.e., it denotes direct product of $k$ copies of the cyclic group of order $n$.

$A . B$ denotes any group having a normal subgroups isomorphic to $A$ and corresponding factor group isomorphic to $B$.

$A: B \quad$ is an instance of $A . B$ when the extension is split.

$A \cdot B \quad$ is the case of $A . B$ when the extension is not split.

$p^{m+n} \quad$ is a case of $p^{m} \cdot p^{n}$.

$S_{n} \quad$ is the symmetric group of degree of $n$.

$A_{n} \quad$ is the alternating group of degree $n$.

$D_{n} \quad(n$ even) is the dihedral group of order $n$.

$Q_{8} \quad$ is the quaternion group.

$W(S) \quad$ is the Weyl group of the simple complex Lie algebra with the Dynkin diagram $S$.

$A *_{C} B$ is the central product of $A$ and $B$ over their common central subgroup $C$. If $C$ is omitted it is a maximal possible such subgroup.

$L_{n}(q) \quad$ is the projective special linear group $P S L_{n}(q)$ over the Galois field $G F(q)$.

$S_{n}(q) \quad\left(n\right.$ even) is the projective symplectic group $P S p_{n}(q)$ over the Galois field $G F(q)$.

$U_{n}(q) \quad$ is the projective special unitary group $P S U_{n}\left(q^{2}\right)$ over the Galois field $G F\left(q^{2}\right)$. 


\section{Presentations of $M_{11}$}

(1.1) Group: $M_{11}$.

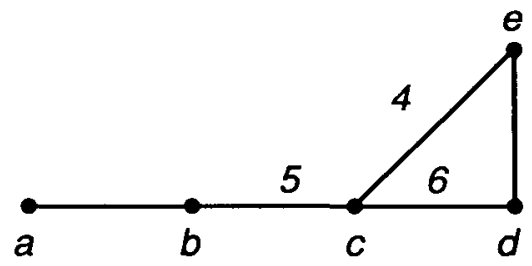

Generators: $a, b, c, d, e$.

Defining relators: $a a, b b, c c, d d, e e, b d b d, b e b e,(a b)^{3},(d e)^{3},(b c)^{5}$, acece, $a(c d)^{3}$.

Length $=64$.

Consequences: acac, adad, aeae, $(c e)^{4},(c d)^{6},(a b c)^{5}$.

Subgroups:

$$
\begin{aligned}
& \langle a, b, c, d\rangle=\langle b, c, d\rangle \cong L_{2}(11),\langle a, b, c, e\rangle=\langle b, c, e\rangle \cong S_{5} \\
& \langle a, c, d, e\rangle=\langle c, d, e\rangle \cong 2 \cdot S_{4} \cong Q_{8}: S_{3},\langle a, b, c\rangle \cong A_{5}
\end{aligned}
$$

Isomorphism:

$$
\begin{array}{ll}
a \rightarrow(0,9)(4,10)(5,8)(6,7), & b \rightarrow(0,2)(1,10)(3,5)(6,7), \\
c \rightarrow(0,7)(1,3)(4,10)(6,9), & d \rightarrow(0,5)(2,3)(6,7)(8,9), \\
e \rightarrow(1,3)(4,8)(5,10)(6,7) . &
\end{array}
$$

(1.2) Group: $M_{11}$.

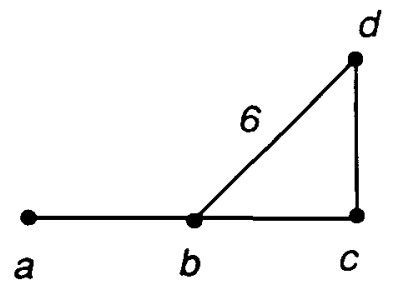

Generators: $a, b, c, d$.

Defining relators: $a a, b b, c c, d d,(a b)^{3},(b c)^{3},(c d)^{3},(a b d b d)^{2},(c b d b d)^{3}$.

Length $=70$.

Consequences: $(b d)^{6}$.

Subgroups:

$$
\langle b, c, d\rangle \cong L_{2}(11),\langle a, b, d\rangle \cong 3^{2}: 2^{2} .
$$


Isomorphism:

$$
\begin{array}{ll}
a \rightarrow(0,7)(1,9)(2,5)(3,6), & b \rightarrow(0,5)(2,3)(6,7)(8,9), \\
c \rightarrow(0,7)(2,6)(3,5)(4,8), & d \rightarrow(0,7)(1,3)(4,10)(6,9) .
\end{array}
$$

(1.3) Group: $M_{11}$.

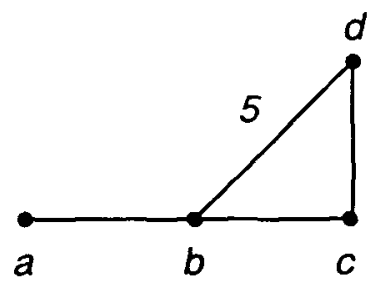

Generators: $a, b, c, d$.

Defining relators: $a a, b b, c c, d d, a c a c, a d a d,(a b)^{3},(b c)^{3},(c d)^{3},(b d)^{5},(a b d)^{5}$, $(b c d b c d c)^{3}$.

Length $=92$.

Subgroups:

$$
\langle b, c, d\rangle \cong L_{2}(11),\langle a, b, d\rangle \cong A_{5} .
$$

Isomorphism:

$$
\begin{array}{ll}
a \rightarrow(0,10)(1,3)(2,5)(4,7), & b \rightarrow(0,2)(1,10)(3,5)(6,7), \\
c \rightarrow(0,5)(1,3)(2,10)(6,8), & d \rightarrow(0,7)(1,3)(4,10)(6,9) .
\end{array}
$$

\section{Presentation of $M_{12}$}

(2.1) Group: $M_{12}$.

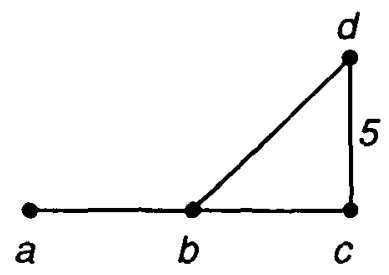

Generators: $a, b, c, d$.

Defining relators: $a a, b b, c c, d d, a c a c, a d a d,(a b)^{3},(b c)^{3},(b d)^{3},(c d)^{5},(b c d b c d c)^{3}$. Length $=76$. 
Subgroups:

$$
\langle b, c, d\rangle \cong L_{2}(11) .
$$

Isomorphism:

$$
\begin{array}{lll}
a \rightarrow(0,8)(1,6)(2, \infty)(3,10)(4,5)(7,9), & b \rightarrow(0, \infty)(1,10)(2,5)(3,7)(4,8)(6,9), \\
c \rightarrow(0,1)(2, \infty)(3,4)(5,10)(6,8)(7,9), & d \rightarrow(0,4)(1,6)(2, \infty)(3,9)(5,8)(7,10) .
\end{array}
$$

As linear fractional transformation over $G F(11)$ we have

$$
b: x \rightarrow-\frac{1}{x}, \quad c: x \rightarrow \frac{2 x-2}{x-2}, \quad d: x \rightarrow \frac{2 x+3}{x-2} .
$$

\section{Presentations of $M_{21}$ and $2^{2} \cdot M_{21}$}

Recall that $M_{21}$ is isomorphic to $L_{3}(4)$.

(3.1) Group: $2^{2} \cdot M_{21}$.

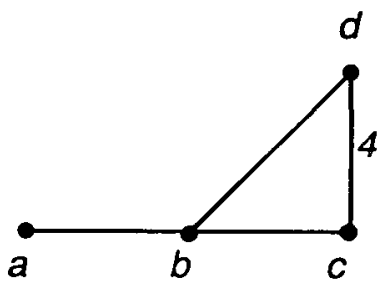

Generators: $a, b, c, d$.

Defining relators: $a a, b b, c c, d d, a c a c, a d a d,(a b)^{3},(b c)^{3},(b d)^{3},(c d)^{4},\left(b c d^{5}\right.$. Length $=68$.

Subgroups:

$$
\langle b, c, d\rangle \cong A_{6} .
$$

The center of $G$ is generated by $(a b c b d)^{5}$ and $(a b c d c d)^{5}$. A homomorphism of $G$ onto $M_{21}$ is given by

$$
\begin{aligned}
& a \rightarrow(2,16)(4,22)(5,9)(7,14)(11,21)(12,20)(15,19)(17,18), \\
& b \rightarrow(4,21)(6,16)(7,13)(8,9)(10,18)(11,15)(12,20)(19,22), \\
& c \rightarrow(2,22)(3,10)(4,16)(5,11)(6,8)(7,14)(9,21)(12,20), \\
& d \rightarrow(2,7)(4,12)(5,18)(9,17)(11,15)(14,16)(19,21)(20,22) .
\end{aligned}
$$


(3.2) If we we add the relators $(a b c b d)^{5}$ and $(a b c d c d)^{5}$ to the above presentation then the relator $(c f)^{4}$ becomes redundant and we obtain a presentation of $L_{3}(4) \cong M_{21}$ of length 116 .

\section{Presentations of $A_{8} \cong L_{4}(2)$}

(4.1) Group: $A_{8}$.

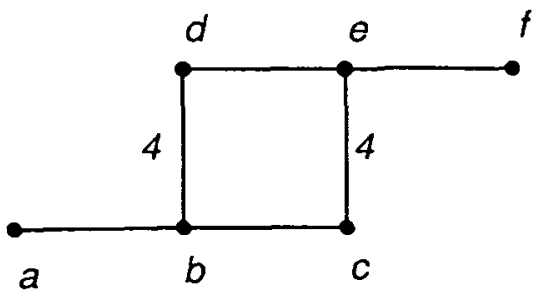

Generators: $a, b, c, d, e, f$.

Defining relators: $a a, b b, c c, d d, e e, f f, a d a d, b e b e, c d c d,(a b)^{3},(b c)^{3},(d e)^{3}$, $(e f)^{3},(b c d)^{3},(c d e)^{3}, f(a b d)^{3}, a(c e f)^{3}$.

Length $=103$.

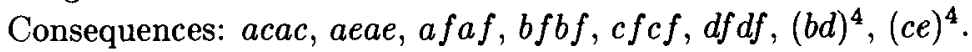

Subgroups:

$$
\langle a, b, c, e, f\rangle \cong\langle a, b, d, e, f\rangle \cong 2^{4}:\left(S_{3} \times S_{3}\right),\langle b, c, d, e\rangle \cong 2^{3}: S_{4} .
$$

Isomorphism:

$$
\begin{array}{ll}
a \rightarrow(1,4)(2,7)(3,8)(5,6), & b \rightarrow(1,2)(3,7)(4,8)(5,6) \\
c \rightarrow(1,3)(2,5)(4,8)(6,7), & d \rightarrow(1,4)(2,6)(3,8)(5,7), \\
e \rightarrow(1,2)(3,8)(4,7)(5,6), & f \rightarrow(1,3)(2,7)(4,8)(5,6) .
\end{array}
$$

(4.2) Group: $A_{8}$.

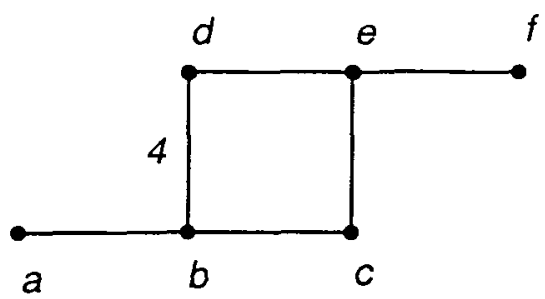

Generators: $a, b, c, d, e, f$.

Defining relators: $a a, b b, c c, d d, e e, f f, b e b e, c d c d, c f c f, d f d f,(a b)^{3}(b c)^{3}$, $(c e)^{3},(d e)^{3},(e f)^{3},(b c d)^{3}, f(a b d)^{3}, a(c d e f)^{3}$. 
Length $=108$.

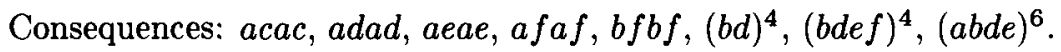
Subgroups:

$$
\begin{aligned}
& \langle a, b, c, e, f\rangle \cong S_{6},\langle a, b, d, e, f\rangle \cong 2^{4}:\left(S_{3} \times S_{3}\right), \\
& \langle b, c, d, e\rangle \cong 2^{3}: L_{3}(2),\langle c, e, b d b d\rangle \cong L_{3}(2)
\end{aligned}
$$

Isomorphism: The images of $a, b, d, e, f$ are the same as in (4.1) above;

$$
c \rightarrow(1,8)(2,7)(3,4)(5,6) .
$$

5. Presentations of $M_{22}, 2 \cdot M_{22}$, and $6 \cdot M_{22}$

(5.1) Group: $M_{22}$.

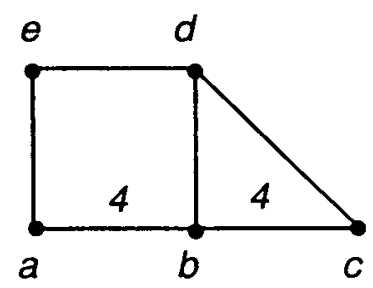

Generators: $a, b, c, d, e$.

Defining relators: $a a, b b, c c, d d, e e, a c a c, a d a d, b e b e, c e c e,(a e)^{3},(b d)^{3},(c d)^{3}$, $(d e)^{3},(b c)^{4},(a b d)^{3},(a b e)^{3},(b c d)^{5}$.

Length $=108$.

Consequences: $(a b)^{4}$.

Subgroups:

$$
\begin{aligned}
& \langle a, b, c, d\rangle \cong 2^{4}: A_{6},\langle b, c, d, e\rangle \cong M_{21} \cong L_{3}(4), \\
& \langle b, c, d\rangle \cong A_{6},\langle a, b, d, e\rangle \cong L_{3}(2) \cong L_{2}(7) \\
& \langle a, c, d, e\rangle \cong S_{5},\langle a, b, c, e\rangle \cong 2^{3}: S_{4}
\end{aligned}
$$

Isomorphism:

$$
\begin{aligned}
& a \rightarrow(1,3)(2,14)(4,22)(6,13)(7,16)(11,15)(12,20)(19,21), \\
& b \rightarrow(2,7)(3,11)(4,20)(5,10)(6,21)(8,9)(12,16)(14,22), \\
& c \rightarrow(2,16)(4,22)(5,9)(7,14)(11,21)(12,20)(15,19)(17,18), \\
& d \rightarrow(2,22)(3,10)(4,16)(5,11)(6,8)(7,14)(9,21)(12,20), \\
& e \rightarrow(4,21)(6,16)(7,13)(8,9)(10,18)(11,15)(12,20)(19,22) .
\end{aligned}
$$


(5.2) Group: $2 \cdot M_{22}$.

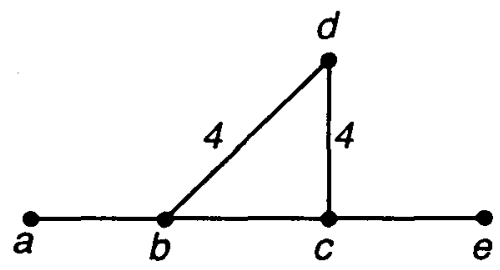

Generators: $a, b, c, d, e$.

Defining relators; $a a, b b, c c, d d, a c a c$, adad $,(a b)^{3},(b c)^{3},(c e)^{3},(b d)^{4},(c d)^{4}$, $(c d e)^{3}, e(a b d)^{3},(b c d e)^{5}$.

Length $=103$.

Consequences: ee, aeae, bebe, dede.

Subgroups:

$$
\begin{aligned}
& \langle a, b, c, e,\rangle \cong S_{5},\langle b, c, d, e\rangle=\langle b, c, d\rangle \cong M_{21}, \\
& \langle a, b, d, e\rangle=\langle a, b, d\rangle \cong 2 \times S_{4},\langle c, d, e\rangle \cong S_{4} .
\end{aligned}
$$

Homomorphism onto $M_{22}$ is given by

$$
\begin{aligned}
& a \rightarrow(1,3)(2,14)(4,22)(6,13)(7,16)(11,15)(12,20)(19,21), \\
& b \rightarrow(2,22)(3,10)(4,16)(5,11)(6,8)(7,14)(9,21)(12,20), \\
& c \rightarrow(4,21)(6,16)(7,13)(8,9)(10,18)(11,15)(12,20)(19,22), \\
& d \rightarrow(2,14)(4,20)(5,17)(7,16)(9,18)(11,19)(12,22)(15,21), \\
& e \rightarrow(2,16)(4,22)(5,9)(7,14)(11,21)(12,20)(15,19)(17,18) .
\end{aligned}
$$

(5.3) Group: $2 \cdot M_{22}$.

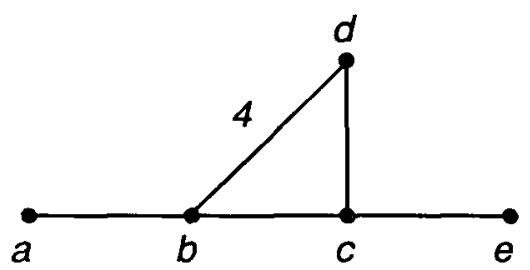

Generators: $a, b, c, d, e$.

Defining relators: $a a, b b, c c, d d, e e, a c a c, a d a d, a e a e, b e b e, d e d e,(a b)^{3},(b c)^{3}$, $(c d)^{3},(c e)^{3},(b d)^{4},(a b d)^{3},(b c d)^{5}$.

Length $=103$.

The center is generated by the element $(b d b d c e)^{5}$. 
Subgroups:

$$
\begin{aligned}
& \langle a, b, c, d\rangle \cong A_{7},\langle b, c, d, e\rangle \cong 2 \cdot M_{21}, \\
& \langle a, b, c, e\rangle \cong S_{5},\langle b, c, d\rangle \cong A_{6} .
\end{aligned}
$$

Homomorphism onto $M_{22}$ sends $a, b, c, e$ to permutations given in (5.2) above while

$$
d \rightarrow(2,7)(4,12)(5,18)(9,17)(11,15)(14,16)(19,21)(20,22) .
$$

(5.4) Group: $6 \cdot M_{22}$.

Diagram: Same as the diagram in (1.3).

Generators: $a, b, c, d$.

Defining relators: $a a, b b, c c, d d, a c a c, a d a d,(a b)^{3},(b d)^{3},(c d)^{3},(b d)^{5},(a b d)^{5}$, $(a b c d b)^{4}$.

Length $=91$.

The center is generated by $(a b d c b d)^{5}$.

Subgroups:

$$
\langle a, b, d\rangle \cong A_{5} .
$$

Homomorphism onto $M_{22}$ is given by:

$$
\begin{aligned}
& a \rightarrow(1,3)(2,14)(4,22)(6,13)(7,16)(11,15)(12,20)(19,21), \\
& b \rightarrow(2,22)(3,10)(4,16)(5,11)(6,8)(7,14)(9,21)(12,20), \\
& c \rightarrow(4,21)(6,16)(7,13)(8,9)(10,18)(11,15)(12,20)(19,22), \\
& d \rightarrow(1,16)(3,7)(4,22)(5,8)(10,17)(11,15)(12,21)(19,20) .
\end{aligned}
$$

\section{Presentations of $M_{23}$}

(6.1) Group: $M_{23}$.

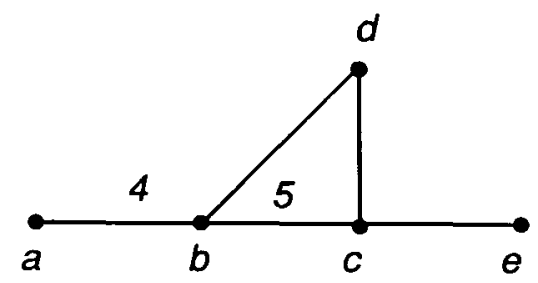

Generators: $a, b, c, d, e$.

Defining relators: $a a, b b, c c, d d, a c a c, a d a d,(b d)^{3},(c d)^{3},(c e)^{3},(a b)^{4},(b c)^{5}$, $e(a b d)^{3},(b c e)^{5},(a b a b c)^{3},(b c d b c d c)^{3}$.

Length $=128$.

Consequences: ee, aeae, bebe, dede, $(a b c)^{6},(b c d)^{11}$. 
Subgroups:

$$
\begin{aligned}
& \langle b, c, d, e\rangle \cong M_{11},\langle a, b, c, e\rangle \cong 2^{4}: S_{5} \\
& \langle b, c, d\rangle \cong L_{2}(11),\langle a, b, c\rangle \cong S_{5},\langle b, c, e\rangle \cong A_{5} .
\end{aligned}
$$

Isomorphism:

$$
\begin{aligned}
& a \rightarrow(1,13)(4,22)(5,15)(7,14)(8,10)(9,19)(11,17)(18,21), \\
& b \rightarrow(0,11)(1,12)(3,13)(4,22)(5,14)(6,20)(10,21)(16,17), \\
& c \rightarrow(0,20)(1,13)(2,3)(4,5)(8,11)(10,17)(15,22)(18,21), \\
& d \rightarrow(1,13)(3,6)(4,22)(5,18)(9,17)(11,19)(12,20)(15,21), \\
& e \rightarrow(1,13)(3,12)(4,22)(5,21)(6,20)(7,8)(10,14)(15,18) .
\end{aligned}
$$

(6.2) Group: $M_{23}$.

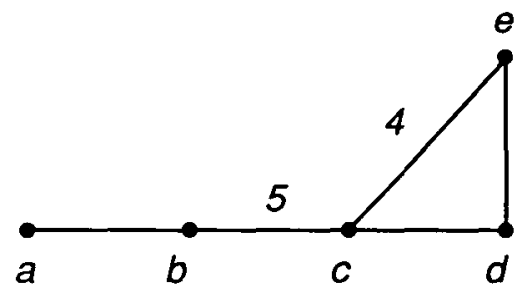

Generators: $a, b, c, d, e$.

Defining relators: $a a, b b, c c d d, e e, a c a c, a d a d, a e a e, b d b d, b e b e,(a b)^{3},(c d)^{3}$, $(d e)^{3},(c e)^{4},(b c)^{5},(b c d)^{5},(b c e)^{5},(d c e c e)^{3}$.

Length $=129$.

Consequences: $(a b c)^{5},(b c d e)^{7},(b c d e d)^{6}$.

Subgroups:

$$
\begin{aligned}
& \langle a, b, c, e\rangle \cong 2^{4}: A_{5},\langle b, c, d, e\rangle \cong M_{22}, \\
& \langle a, b, c, d\rangle \cong L_{2}(11),\langle a, b, c\rangle \cong\langle b, c, d\rangle \cong A_{5} \\
& \langle b, c, e\rangle \cong 2^{4}: D_{10},\langle c, d, e\rangle \cong L_{3}(2) \cong L_{2}(7) .
\end{aligned}
$$

Isomorphism:

$$
\begin{aligned}
& a \rightarrow(1,22)(2,15)(3,13)(4,12)(6,20)(8,9)(10,17)(16,21), \\
& b \rightarrow(0,20)(1,13)(2,3)(4,5)(8,11)(10,17)(15,22)(18,21), \\
& c \rightarrow(0,11)(1,12)(3,13)(4,22)(5,14)(6,20)(10,21)(16,17), \\
& d \rightarrow(0,18)(1,2)(3,13)(6,16)(7,14)(10,17)(15,22)(20,21), \\
& e \rightarrow(1,22)(2,3)(4,21)(5,18)(10,17)(12,16)(13,15)(14,19) .
\end{aligned}
$$

(6.3) Group: $M_{23}$.

Diagram: Same as in (6.1) above. 
Generators: $a, b, c, d, e$.

Defining relators: $a a, b b, c c, d d, e e, a c a c$, adad, aeae, bebe, dede, $(b d)^{3},(c d)^{3}$, $(c e)^{3},(b c)^{5},(a b d)^{3},(b c d)^{5},(b c e)^{5},(a b a b c)^{3}$.

Length $=130$.

Consequences: $(a b)^{4}$.

Subgroups:

$$
\begin{aligned}
& \langle a, b, c, d\rangle \cong M_{11},\langle b, c, d, e\rangle \cong 2^{4}: A_{7}, \\
& \langle a, b, c, e\rangle \cong 2^{4}: S_{5},\langle a, b, d\rangle \cong S_{4}, \\
& \langle a, b, c\rangle \cong S_{5},\langle b, c, d\rangle \cong A_{6},\langle b, c, e\rangle \cong A_{5} .
\end{aligned}
$$

Isomorphism: The images of $a, b, c, e$ are the same as in (6.1) above;

$$
d \rightarrow(1,4)(3,12)(5,15)(6,20)(9,11)(13,22)(17,19)(18,21) .
$$

(6.4) Group: $M_{23}$.

Diagram: Same is in (6.2) above.

Generators: $a, b, c, d, e$.

Defining relators: $a a, b b, c c, d d, e e, a c a c, a d a d, a e a e, b d b d, b e b e,(a b)^{3},(c d)^{3}$, $(d e)^{3},(c e)^{4},(b c)^{5},(b c d)^{5},(d c e c e)^{3},(b c d e d)^{5}$.

Length $=139$.

Consequences: $(a b c)^{5},(b c e)^{6},(b c d e)^{7}$.

Subgroups:

$$
\begin{aligned}
& \langle a, b, c, d\rangle \cong L_{2}(11),\langle a, b, c, e\rangle \cong 2^{4}: A_{5} \\
& \langle b, c, d, e\rangle \cong M_{22},\langle c, d, e\rangle \cong L_{3}(2) \cong L_{2}(7), \\
& \langle a, b, c\rangle \cong\langle b, c, d\rangle \cong A_{5},\langle b, c, e\rangle \cong S_{5}
\end{aligned}
$$

Isomorphism: The images of $a, b, c, d$ are the same in (6.2) above;

$$
e \rightarrow(0,5)(1,13)(2,15)(3,22)(4,20)(6,12)(7,19)(10,17) .
$$

\section{Presentation of $M_{24}$}

(7.1) Group: $M_{24}$.

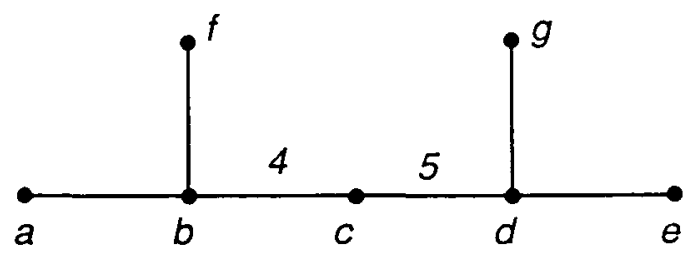


Generators: $a, b, c, d, e, f, g$.

Defining relators: $b b, c c, d d, e e, f f, a f a f, b d b d, b e b e, c e c e, c f c f, d f d f, e f e f$, $(a b)^{3},(b f)^{3},(d e)^{3},(d g)^{3},(c d)^{5},(b c f)^{3}, g(a b c)^{3}, a(c d e)^{5}$.

Length $=127$.

Consequences: $a a, g g, a c a c, a d a d, a e a e, a g a g, b g b g, c g c g, e g e g, f g f g,(b c)^{4}$, $(c d g)^{5},(d c d g e)^{4},(b c b c d)^{5},(b c d c d f)^{5},(a b c d)^{10}$.

Subgroups:

$$
\begin{aligned}
\langle a, b, c, d, f, g\rangle=\langle a, b, c, d\rangle \cong M_{12}: 2,\langle a, b, c, f\rangle \cong 2^{3}: S_{4}, \\
\langle b, c, d\rangle \cong L_{2}(11): 2,\langle c, d, e\rangle \cong 2 \times A_{5},\langle c, d, g\rangle \cong A_{5} \\
\langle b, c, f\rangle \cong S_{4},\langle a, b, c\rangle \cong 2 \times S_{4} . \\
a \rightarrow(0,4)(1,6)(2, \infty)(3,21)(5,16)(7,15)(8,9)(10,13) \\
\quad(11,22)(12,20)(14,17)(18,19), \\
b \rightarrow(0,4)(1,5)(2, \infty)(3,20)(6,13)(7,9)(8,18)(10,16) \\
\quad(11,22)(12,14)(15,19)(17,21), \\
c \rightarrow(0, \infty)(1,13)(2,4)(3,8)(5,11)(6,10)(9,21)(16,22), \\
d \rightarrow(1,15)(2,11)(5,19)(6,7)(8,10)(9,13)(16,18)(22, \infty), \\
e \rightarrow(0,2)(3,9)(4, \infty)(7,20)(8,21)(12,15)(14,19)(17,18), \\
f \rightarrow(0,3)(1,5)(2,9)(4,21)(6,16)(7,18)(8, \infty)(10,22) \\
\quad(11,13)(12,14)(15,19)(17,20), \\
g \rightarrow(0, \infty)(2,4)(3,8)(7,17)(9,21)(12,19)(14,15)(18,20) .
\end{aligned}
$$

\section{Presentation of $J_{1}$}

(8.1) Group: $J_{1}$.

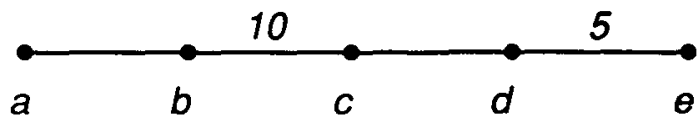

Generators: $a, b, c, d, e$.

Defining relators: $b b, c c, d d, e e, b d b d,(a b)^{3},(c d)^{3},(d e)^{5}, e(b c)^{5}, a(c d e)^{5}$, $e(a b c)^{5}$.

Length $=88$.

Consequences: aa, acac, adad, aeae, bebe, cece, $(b c)^{10}$. 
Subgroups:

$$
\begin{aligned}
& \langle a, b, c, e\rangle=\langle a, b, c\rangle \cong\langle a, c, d, e\rangle=\langle c, d, e\rangle \cong 2 \times A_{5}, \\
& \langle a b c d, e d e\rangle \cong L_{2}(11) .
\end{aligned}
$$

\section{Presentation of $J_{2}$}

(9.1) Group: $J_{2}$.

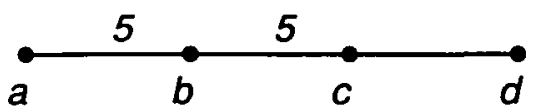

Generators: $a, b, c, d$.

Defining relators: $a a, b b, c c, d d, a c a c, a d a d, b d b d,(c d)^{3},(a b)^{5},(b c)^{5},(b c d)^{5}$, $\left(a b c(a b c b)^{2}\right)^{2}$.

Length $=95$.

Consquences: $(a b c)^{6}$.

Subgroups:

$$
\langle a, b, c\rangle \cong A_{5} \times D_{10},\langle b, c, d\rangle \cong A_{5}
$$

\section{Presentations of $H S$}

(10.1) Groups: HS.

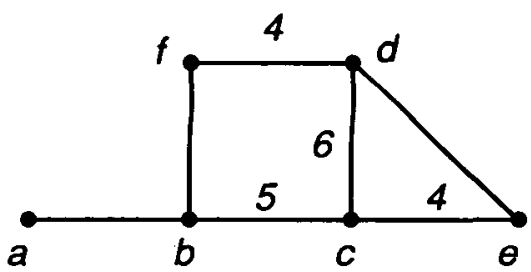

Generators: $a, b, c, d, e, f$.

Defining relators: $b b, c c, d d, e e, f f, b d b d, b e b e, c f c f, e f e f,(a b)^{3},(b f)^{3},(d e)^{3}$, $(b c)^{5}, a c e c e, a(c d)^{3}, a(c d f)^{3}, a(d e f)^{3},(b c f)^{5}$.

Length $=119$.

Consequences: aa, acac, adad, aeae, afaf, $(c e)^{4},(d f)^{4},(c d)^{6},(a b c)^{5},(a b f d)^{4}$. 
Subgroups:

$$
\begin{aligned}
& \langle a, b, c, d, e\rangle \cong M_{11},\langle a, b, c, d, f\rangle=\langle b, c, d, f\rangle \cong M_{22} \\
& \langle a, b, d, e, f\rangle \cong W\left(F_{4}\right) / 2,\langle a, b, c, e, f\rangle \cong 2^{4}: S_{5} \\
& \langle a, b, c, f\rangle \cong 2^{4}: A_{5},\langle a, b, c, d\rangle \cong L_{2}(11) \\
& \langle a, b, c, e\rangle=\langle b, c, e\rangle \cong S_{5}
\end{aligned}
$$

(10.2) Group: HS.

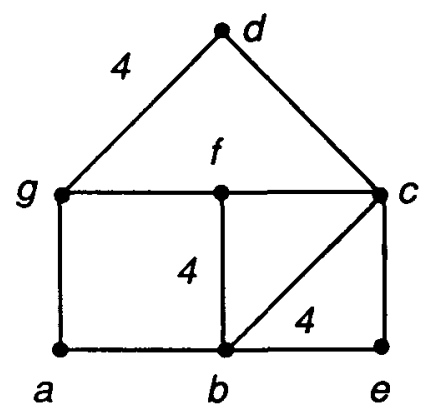

Generators: $a, b, c, d, e, f, g$.

Defining relators: $a a, b b, c c, d d, e e, f f, g g, a c a c, a f a f, b g b g, c g c g,(a b)^{3}$, $(a g)^{3},(b c)^{3},(c d)^{3},(c f)^{3},(f g)^{3},(a b f)^{3},(b f g)^{3}, d(b e b f)^{2}, d(a b e)^{3}, e(a d g)^{3}$, $a(c d e f)^{3},(b c f)^{5}$.

Length $=165$.

Consequences: adad, aeae, bdbd, dede, dfdf, efef, egeg, $(c e)^{3},(b e)^{4},(b f)^{4}$, $(d g)^{4},(d f g)^{3}$.

Subgroups:

$$
\begin{aligned}
& \langle a, b, c, d, e, f\rangle=\langle b, c, e, f\rangle \cong\langle b, c, d, f, g\rangle \cong M_{22} \\
& \langle a, b, c, d, e, g\rangle=\langle a, b, c, d, g\rangle=\langle a, b, c, e, g\rangle \cong 2^{4}: S_{6} \\
& \langle a, b, c, d, e\rangle=\langle a, b, c, e\rangle \cong 2^{4}: S_{5} \\
& \langle a, b, c, f, g\rangle \cong U_{3}(5),\langle a, b, c, f\rangle \cong\langle b, c, f, g\rangle \cong A_{7} \\
& \langle a, c, d, e, f, g\rangle=\langle a, c, d, f, g\rangle=\langle c, d, e, f, g\rangle \cong M_{21} \cong L_{3}(4) \\
& \langle a, c, d, e, g\rangle=\langle a, c, d, g\rangle \cong W\left(F_{4}\right) / 2 \\
& \langle b, c, f\rangle \cong A_{6},\langle b, c, d, f\rangle \cong M_{21} \cong L_{3}(4) \\
& \langle c, d, f, g\rangle \cong\langle a, b, d, e, f, g\rangle=\langle a, b, d, f, g\rangle \cong 2^{3}: L_{3}(2) \\
& \langle b, c, d, e, g\rangle \cong\left(2 \times 4^{2}\right): S_{4},\langle b, c, d, e\rangle \cong 2^{4}: S_{4} \\
& \langle b, c, e,\rangle \cong 4^{2}: S_{3},\langle a, b, f, g\rangle \cong L_{3}(2) \cong L_{2}(7) \\
& \langle a, b, d, e, g\rangle \cong\langle b, d, e, f, g\rangle=\langle b, e, f, g\rangle \cong 2^{3}: S_{4} \\
& \langle a, c, e, f, g\rangle \cong S_{6},\langle a, b, c, g\rangle \cong S_{5},\langle b, e, f\rangle \cong 2^{3}: D_{8}
\end{aligned}
$$




$$
\begin{aligned}
& \langle a, d, f, g\rangle \cong\langle a, b, e, f\rangle \cong\langle c, d, e, f\rangle \cong 2^{3}: S_{4}, \\
& \langle a, b, e\rangle \cong\langle a, d, g\rangle \cong\langle c, d, g\rangle \cong 2^{3}: S_{3},\langle a, b, f\rangle \cong S_{4} .
\end{aligned}
$$

\section{Presentations of $M c L$}

(11.1) Group: $M c L$.

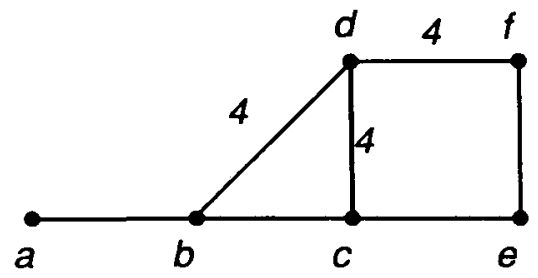

Generators: $a, b, c, d, e, f$.

Defining relators: $a a, b b, c c, d d, e e, f f, b e b e, b f b f, c f c f, d e d e,(a b)^{3},(b c)^{3}$, $(c e)^{3},(e f)^{3}, a(c d f d)^{2}, a(d e f)^{3}, e(a b d)^{3},(b c d e)^{5}$.

Length $=119$.

Consequences: acac, adad, aeae, afaf, $(b d)^{4},(c d)^{4},(d f)^{4},(c d e)^{3},(b c b d)^{5}$, $(b c d)^{7}$.

Subgroups:

$$
\begin{aligned}
& \langle a, b, c, d, e\rangle=\langle a, b, c, d\rangle \cong M_{22} \\
& \langle b, c, d, e\rangle=\langle b, c, d\rangle \cong M_{21} \cong L_{3}(4) \\
& \langle a, b, d, e\rangle=\langle a, b, d\rangle \cong\langle a, d, e, f\rangle=\langle d, e, f\rangle \cong 2 \times S_{4} \\
& \langle a, b, c, e, f\rangle \cong\langle a, b, d, e, f\rangle \cong S_{6},\langle c, d, e,\rangle \cong S_{4} \\
& \langle a, c, d, e, f\rangle=\langle c, d, e, f\rangle \cong 2^{4}: S_{4} \\
& \langle b, d, f\rangle \cong 3^{2}: D_{8},\langle c, d, f\rangle \cong\left(D_{8} * D_{8}\right): 2
\end{aligned}
$$

(11.2) Group: $M c L$.

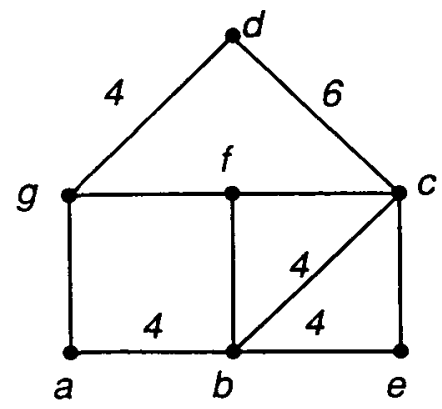


Generators: $a, b, c, d, e, f, g$.

Defining relators: $a a, b b c c, d d, e e, f f, g g, a e a e, a f a f, b g b g, e f e f,(a g)^{3},(b f)^{3}$, $(c e)^{3},(c f)^{3},(f g)^{3},(d g)^{4}, a(c d)^{3},(a b f)^{3}, d(a b e b)^{2}, d(b e f)^{3}, e(d g f)^{3},(a b c d)^{3}$, $g(b c e c)^{3}$.

Length $=162$.

Consequences: acac, adad, bdbd, cgcg, dede, dfdf, egeg, $(a b)^{4},(b c)^{4},(b e)^{4}$, $(c d)^{6},(a b g)^{3},(a d g)^{3},(c d e f)^{3},(c d g d)^{3},(a b c)^{4},(a b e)^{4},(c d e)^{4},(c d f)^{4},(b c f)^{5}$, $(c d g)^{5},(a b f c)^{5},(b c d e)^{5},(b c e)^{7},(b c d b f)^{5}$.

Subgroups:

$$
\begin{aligned}
& \langle a, b, c, f, g\rangle \cong M_{22}, \\
& \langle a, b, c, d, e, g\rangle \cong\langle a, b, c, e\rangle \cong M_{21}: 2 \cong L_{3}(4): 2 \\
& \langle b, c, f, g\rangle \cong M_{21},\langle a, b, c, d, f\rangle \cong 2^{4}: A_{7} \\
& \langle a, c, d, e, f, g\rangle=\langle c, d, f, g\rangle \cong U_{4}(2) \cong S_{4}(3) \\
& \langle a, b, c, d, g\rangle=\langle b, c, d, g\rangle \cong 2^{4}: S_{5} \\
& \langle a, c, e, f, g\rangle \cong S_{6},\langle a, b, c, f\rangle \cong 2^{4}: A_{6} \\
& \langle a, b, d, e, f, g\rangle=\langle a, b, d, f, g\rangle=\langle a, b, e, f, g\rangle \cong 2^{3}: L_{3}(2) \\
& \langle a, b, c\rangle \cong\langle a, b, d, e\rangle=\langle a, b, e\rangle \cong\left(D_{8} * D_{8}\right): 2, \\
& \langle a, b, f, g\rangle \cong L_{3}(2) \cong L_{2}(7) \\
& \langle a, c, d, e, g\rangle \cong S_{6},\langle b, c, f\rangle \cong A_{6}, \\
& \langle b, c, e, g\rangle=\langle b, c, e\rangle \cong 2 \times L_{3}(2) \cong 2 \times L_{2}(7) \\
& \langle a, b, d, g\rangle \cong 2^{4}: S_{3},\langle a, c, d, g\rangle \cong S_{5}, \\
& \langle a, c, d, e, f\rangle=\langle c, d, e, f\rangle \cong\langle a, b, c, d\rangle=\langle b, c, d\rangle \cong 2^{3}: S_{4}, \\
& \langle b, d, e, f, g\rangle=\langle b, e, f, g\rangle \cong\langle a, b, d, e, f\rangle=\langle a, b, e, f\rangle \cong 2^{3}: S_{4} \\
& \langle a, b, d, e, g\rangle=\langle a, b, e, g\rangle \cong\langle a, b, c, g\rangle \cong 2^{3}: S_{4}, \\
& \langle a, b, g\rangle \cong\langle a, d, g\rangle \cong\langle a, b, f\rangle \cong S_{4}, \\
& \langle b, d, e, f\rangle \cong\langle d, g, f\rangle \cong 2 \times S_{4} .
\end{aligned}
$$

\section{Presentations of $S_{6}(2)$ and related groups}

The group $2 \cdot S_{6}(2)$ is a centralizer of an involution in the smallest Conway group $\mathrm{Co}_{3}$. All presentations in this section were discovered while searching for a simple presentation of $\mathrm{Co}_{3}$. The group $2 \times S_{6}(2)$ is isomorphic to the Weyl group of the simple complex Lie algebra of type $E_{7}$. As such it has a Coxeter 
type presentation of length 138 . We give here a shorter presentation for that group.

(12.1) Group: $2 \times S_{6}(2) \cong W\left(E_{7}\right)$.

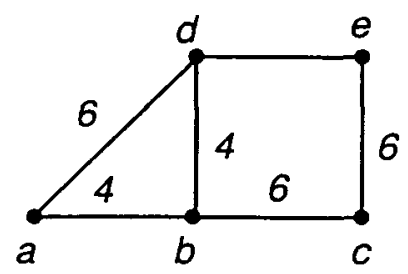

Generators: $a, b, c, d, e$.

Defining relators: $a a, b b, c c, d d, e e, b e b e,(d e)^{3}, a(c e)^{3}, c(a d)^{3},(b d e)^{3},(b c e)^{4}$, $(a b a b d)^{3}$.

Length $=92$.

Subgroups:

$$
\begin{aligned}
& \langle a, b, c, d\rangle=\langle a, b, d\rangle \cong U_{3}(3): 2, \\
& \langle a, b, c, e\rangle=\langle b, c, e\rangle \cong 2^{4}: D_{12}, \\
& \langle a, c, d, e\rangle=\langle a, d, e\rangle=\langle c, d, e\rangle \cong\left(3^{2}: 3\right): 2^{2}, \\
& \langle a, b, c\rangle \cong 2 \times S_{4},\langle b, d, e\rangle \cong S_{4} .
\end{aligned}
$$

(12.2) By adding the relator $(a b d e)^{7}$ to the presentation (12.1) we obtain a presentation of $S_{6}(2)$ of length 121 .

(12.3) Group: $S_{6}(2)$.

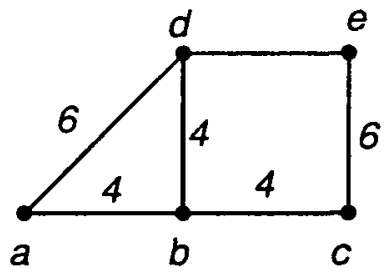

Generators: $a, b, c, d, e$.

Defining relators: $a a, b b, c c, d d, e e, b e b e,(d e)^{3},(b c)^{4},(b d)^{4} a(c e)^{3}, c(a d)^{3}$, $(b d e)^{3},(a b a d)^{3},(a b a b d)^{3},(a b d)^{7}$.

Length $=122$.

Subgroups:

$$
\begin{aligned}
& \langle a, b, c, d\rangle=\langle a, b, d\rangle \cong 2^{6}: L_{3}(2) \\
& \langle a, b, c, e\rangle=\langle b, c, e\rangle \cong 2^{4}: D_{12},\langle b, c, d\rangle \cong 2^{4}: D_{8} \\
& \langle a, c, d, e\rangle=\langle a, d, e\rangle=\langle c, d, e\rangle \cong\left(3^{2}: 3\right): 2^{2}
\end{aligned}
$$


(12.4) Group: $2 \cdot S_{6}(2)$.

Diagram: It is the sub-diagram of the diagram in (13.1) which is obtained by deleting the vertex $d$ and the two incident edges.

Generators: $a, b, c, e, f, g$.

Defining relators: $a a, b b, c c, e e, f f, g g, a c a c$, aeae, cece, egeg, $(a b)^{3}, f(a g)^{3}$, $g(b f)^{3},(c f g)^{3}, e(a b c)^{3}, e(a c g)^{4},(b c f c f)^{3}, e(a b c f)^{7}$.

Length $=142$.

Consequences: afaf, efef, $f g f g,(b c)^{4},(c f)^{4},(a g)^{6},(c g)^{6}$.

Subgroups: See presentation (13.1) where this group occurs as a subgroup.

(12.5) By setting $e=1$ in the presentation (12.4) we obtain yet another presentation of $S_{6}(2)$ of length 121 .

(12.6) By omitting the defining relator $e(a b c f)^{7}$ in the presentation (12.4) we obtain a presentation of $2 \times 2 \cdot S_{6}(2)$ of length 112 .

\section{Presentations of $\mathrm{Co}_{3}$}

(13.1) Group: $\mathrm{Co}_{3}$.

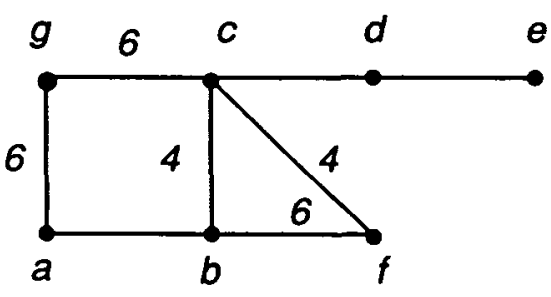

Generators: $a, b, c, d, e, f, g$.

Defining relators: $a a, b b, c c, d d, e e, f f, g g, a c a c, a d a d, a e a e, b d b d, c e c e$, $d g d g, e g e g,(a b)^{3},(c d)^{3},(d e)^{3}, f(a g)^{3}, g(b f)^{3},(c f g)^{3}, e(a b c)^{3}, e(a c g)^{4},(b c f c f)^{3}$, $e(a b c f)^{7}$.

Length $=174$.

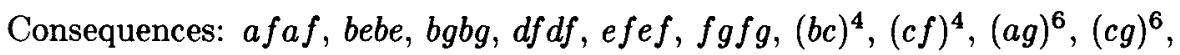
$a(c d e f)^{4},(b c d e)^{4}$. 
Subgroups:

$$
\begin{aligned}
& \langle b, c, d, f, g\rangle=\langle b, c, d, f\rangle \cong\langle b, c, d, e, g\rangle \cong M c L: 2 \\
& \langle a, b, c, e, f, g\rangle=\langle a, b, c, f\rangle=\langle a, b, c, g\rangle \cong 2 \cdot S_{6}(2) \\
& \langle a, c, d, e, f, g\rangle=\langle a, c, d, g\rangle=\langle c, d, e, f, g\rangle \cong U_{4}(2): 2 \cong S_{4}(3): 2, \\
& \langle b, c, f, g\rangle=\langle b, c, f\rangle \cong U_{3}(3): 2 \\
& \langle a, b, c, d, e\rangle=\langle a, b, c, d\rangle \cong W\left(F_{4}\right) / 2 \\
& \langle b, c, d, g\rangle \cong U_{4}(3): 2,\langle c, d, e, g\rangle \cong\langle c, d, f, g\rangle \cong 3^{3}:\left(2 \times S_{4}\right) \\
& \langle a, b, f, g\rangle=\langle a, b, f\rangle=\langle a, b, g\rangle \cong\langle c, d, g\rangle \cong\left(3^{2}: 3\right): 2^{2} \\
& \langle c, f, g\rangle \cong 2 \times S_{4},\langle b, c, g\rangle \cong L_{3}(2): 2 .
\end{aligned}
$$

(13.2) Group: $\mathrm{Co}_{3}$.

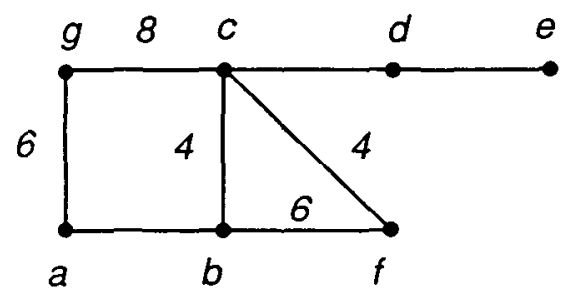

Generators: $a, b, c, d, e, f, g$.

Defining relators: $a a, b b, c c, d d, f f, g g, a c a c, a d a d, b d b d, d f d f,(a b)^{3},(c d)^{3}$, $(d e)^{3},(b c)^{4}, f(a g)^{3}, g(b f)^{3}, e(c g)^{4}, e(a b c)^{3},(b f c f)^{3},(b c f c f)^{3}, a(c d e f)^{4}, e(b c f)^{7}$.

Length $=175$.

Consequences: ee, aeae, afaf, bebe, bgbg, cece, dgdg, efef, egeg, $f g f g,(c f)^{4}$, $(a g)^{6},(b f)^{6},(c g)^{8},(b c d e)^{4}$.

Subgroups:

$$
\begin{aligned}
& \langle a, b, c, e, f, g\rangle=\langle a, b, c, f\rangle=\langle a, b, c, g\rangle \cong 2 \cdot S_{6}(2) \\
& \langle a, c, d, e, f, g\rangle=\langle a, c, d, e, g\rangle=\langle c, d, e, f, g\rangle \cong S_{8} \\
& \langle a, b, c, d, e\rangle=\langle a, b, c, d\rangle \cong W\left(F_{4}\right) / 2 \\
& \langle b, c, e, f, g\rangle=\langle b, c, f\rangle \cong 2^{1+6}: L_{3}(2) \\
& \langle c, e, f, g\rangle=\langle c, f, g\rangle \cong 2^{4}: D_{4} \\
& \langle b, c, e, g\rangle=\langle b, c, g\rangle \cong\left(D_{8} * Q_{8}\right): D_{8} \\
& \langle a, b, f, g\rangle=\langle a, b, f\rangle=\langle a, b, g\rangle \cong\left(3^{2}: 3\right): 2^{2} \\
& \langle c, d, e, g\rangle=\langle c, d, g\rangle \cong L_{3}(2): 2
\end{aligned}
$$


(13.3) Group: $\mathrm{Co}_{3}$.

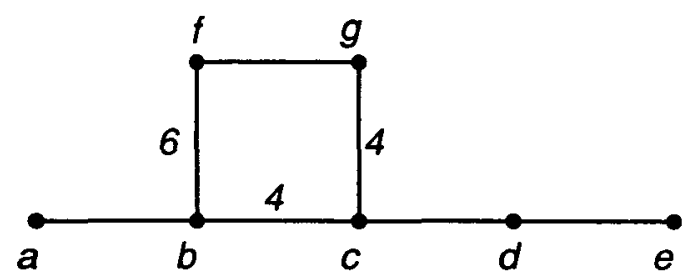

Generators: $a, b, c, d, e, f, g$.

Defining relators: $a a, b b, c c, d d, e e, f f, g g, a d a d, a f a f, a g a g, b d b d, b g b g$, $c f c f, d f d f, d g d g,(a b)^{3},(c d)^{3},(b f b f g)^{2}, e(a b c)^{3}, a e(c f g)^{3}, e(b c g)^{4},(b c b f b f)^{3}$, $e(b c f g f)^{7}$.

Length $=179$.

Consequences: acac, aeae, bebe cece, efef, egeg, $(d e)^{3},(f g)^{3},(b c)^{4},(c g)^{4}$, $(b f)^{6},(b c d e)^{4}, a(c d e g)^{4}, a(c d f g)^{4}$.

Subgroups:

$$
\begin{aligned}
& \langle a, b, c, d, e, f\rangle=\langle a, b, c, d, f\rangle \cong U_{4}(3): 2^{2} \\
& \langle a, b, c, e, f, g\rangle=\langle b, c, f, g\rangle \cong 2 \cdot S_{6}(2) \\
& \langle a, b, c, d, e, g\rangle=\langle b, c, d, g\rangle \cong S_{8} \\
& \langle a, b, c, e, f\rangle=\langle a, b, c, f\rangle \cong 2 \times U_{2}(3): 2 \\
& \langle b, c, d, e, f\rangle \cong M_{21}: 2^{2} \\
& \langle a, b, c, d, e\rangle=\langle a, b, c, d\rangle \cong W\left(F_{4}\right) / 2 \\
& \langle a, c, d, e, f, g\rangle=\langle c, d, f, g\rangle \cong W\left(F_{4}\right) \\
& \langle a, b, c, e, g\rangle=\langle a, b, c, g\rangle \cong\langle a, c, d, e, g\rangle=\langle c, d, e, g\rangle \cong 2^{4}: S_{4}, \\
& \langle b, c, e, g\rangle=\langle b, c, g\rangle \cong 2^{4}: D_{8}, \\
& \langle a, b, f\rangle \cong\left(3^{2}: 3\right): 2^{2},\langle b, f, g\rangle \cong 3^{2}: 2^{2}, \\
& \langle b, c, e, f\rangle=\langle b, c, f\rangle \cong 2 \times L_{3}(2): 2 \\
& \langle b, c, d, e\rangle \cong 2^{4}: D_{12},\langle a, b, f, g\rangle \cong 3^{3}: D_{12} .
\end{aligned}
$$

14. Presentations of $U_{3}(5), U_{3}(5): 2$, and $4 \cdot U_{4}(3): 2^{2}$

(14.1) Group: $U_{3}(5)$.

Diagram: It is the sub-diagram of the diagram in (10.2) obtained by deleting the nodes $d$ and $e$ and the four incident edges.

Generators : $a, b, c, f, g$. 
Defining relators: $a a, b b, c c, f f, g g, a c a c, a f a f, b g b g, c g c g,(a b)^{3},(a g)^{3}$, $(b c)^{3},(c f)^{3},(f g)^{3},(a b f)^{3},(b f g)^{3},(b c f)^{5}$.

Length $=106$.

Consequences: $(b f)^{4}$.

Subgroups: See sub-section (10.2) where this group occurs as a subgroup.

We now give a presentation of $U_{3}(5): 2$. Since this group is a maximal subgroup of the Rudvalis' simple group $R u$, this presentation might be extendable to a presentation of $R u$.

(14.2) Group: $U_{3}(5): 2$.

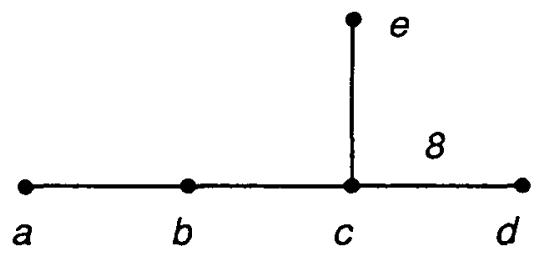

Generators: $a, b, c, d, e$.

Defining relators: $a a, b b, c c, d d, e e, a e a e, b d b d, b e b e, d e d e,(a b)^{3},(b c)^{3},(c e)^{3}$, $a(c d)^{4},(b c d b c d e)^{5}$.

Length $=102$.

Consequences: acac, adad, $(c d)^{8}$.

Subgroups:

$$
\begin{aligned}
& \langle a, b, c, d\rangle=\langle b, c, d\rangle \cong L_{3}(2): 2,\langle a, b, c, e\rangle \cong S_{5}, \\
& \langle a, c, d, e\rangle=\langle c, d, e\rangle \cong\left(4 * Q_{8}\right): S_{3} .
\end{aligned}
$$

(14.3) Group: $4 \cdot U_{4}(3): 2^{2}$.

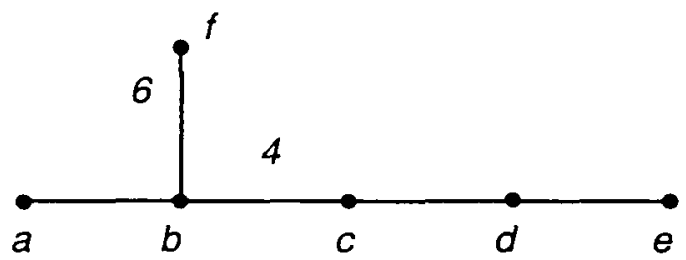

Generators: $a, b, c, d, e, f$.

Defining relators: $a a, b b, c c, d d, f f, a c a c, a d a d, a f a f, b d b d, c f c f, d f d f, e f e f$, $(a b)^{3},(c d)^{3},(b c)^{4},(b f)^{6}, e(a b c)^{3},(a b f)^{6},(b c b f b f)^{3}$.

Length $=135$.

Consequences: ee, aeae, bebe, cece, $(d e)^{6}$. 
Subgroups:

$$
\begin{aligned}
& \langle a, b, c, d, e\rangle=\langle a, b, c, d\rangle \cong 2^{4}:\left(S_{3} \times S_{3}\right) \\
& \langle b, c, d, e, f\rangle \cong 4 \cdot M_{21}: 2^{2} \\
& \langle a, b, c, e, f\rangle=\langle a, b, c, f\rangle \cong 2 \times U_{3}(3): 2
\end{aligned}
$$

\section{Independent verification of some presentations}

(15.1) We first describe the method used in deriving the presentations listed in this paper. We start with a simple group $G$ which is given as a permutation group. For instance the group $\mathrm{Co}_{3}$ has a faithful permutation representation of degree 276. It can be generated by two elements: one involution and another element of order three. We used such a pair of generators from Warboy's paper [8] in order to define in CAYLEY the group $\mathrm{Co}_{3}$ as a permutation group of degree 276. For many other simple groups, for instance the Matheiu groups, $H S$, and $M c L$, such definitions were already available in CAYLEY.

The next most tedious step was to select suitable involutions in $G$ which generate $G$, and to search for simple relations satisfied by these involutions. If $S$ is the set of involutions and $R$ the set of relators that we have selected then we obtain a homomorphism $\phi$ from the abstrac group given by the presentation $\langle S: R\rangle$ onto $G$. With some luck we can show, using coset enumeration, that this homomorphism is an isomorphism.

In the case of Mathieu groups or $A_{8}$ we list the premutations $\phi(S)$ so that the verification of the corresponding presentations is relatively easy. We shall give below the details in the case of the largest Mathieu group $M_{\mathbf{2 4}}$. For Mathieu groups one can use the MOG (the Miracle Octad Generator) or MINI-MOG in order to verify that the listed permutations indeed belong to the corresponding Mathieu group. For that purpose we refer the reader to [2], [3], and [5].

In some other cases one can verify the given presentations by a judicious use of coset enumeration. As an example we shall give below such a verification for the presentation (8.1) of $J_{1}$. All the remaining presentations can be verified by using additional tools: CAYLEY and various characterizations of the relevant simple groups. We give below such a verification of the presentation (10.1) of HS.

(15.2) Let $G=\langle S: R\rangle$ be the group given by the presentation (7.1). We know that there is an epimorphism $\phi: G \rightarrow M_{24}$ as specified in that subsection. Let $K$ be the subgroup of $G$ generated by the elements $a, b, c, d, f$, and $g$. Since $R$ includes the relators: $c c, d d, e e,(d e)^{3},(c d)^{5}$ and $a(c d e)^{5}$, it follows that the relations

$$
a a=a c a c=a \dot{d} a d=1
$$


are valid in $G$. It is easy to show (using coset enumeration) that $g g=1$ is a consequence of the relators: $a a, b b, c c, f f, a c a c, a f a f, c f c f,(a b)^{3},(b f)^{3},(b c f)^{3}$, and $g(a b c)^{3}$. Another coset enumeration (including the redundant relators $a a$ and $g g$ ) shows that $[G: K]=1,288$, that is, it is equal to the index of $M_{12}: 2$ in $M_{24}$.

Now let $H$ be the group with generators $a, b, c, d, f, g$ whose defining relators are acac, adad, and the defining relators of $G$ which involve only generators of $H$. Yet another coset enumeration shows that the order of $H$ is 12 times the order of $M_{12}: 2$.

The above facts imply that the order of $N=\operatorname{Ker} \phi$ divides 12 . Since $G / N \cong$ $M_{24}$, it follows that $N$ is the center of $G$. It is immediate from the presentation of $G$ that it is a perfect group. Since the Schur multiplier of $M_{24}$ is trivial, it follows that $N=\{1\}$, that is, $G \cong M_{24}$.

(15.3) Now let $G$ be the group given by the presentation (8.1), and let $H$ be its subgroup generated by the elements $c, d$, and $e$. A coset enumeration shows that:

$$
[G: H]=1,463=7 \cdot 11 \cdot 19 .
$$

Since $G$ collapses (verified via coset enumeration) when either of the relations $a=1$ or $c d=1$ is added, it follows that $H \cong 2 \times A_{5}$. Consequently the order of $G$ is 175,560 . In order to show that $G \cong J_{1}$, it sufficies to prove that $G$ is simple.

Assume that $G$ has a non-trivial proper normal subgroup $N$. The facts mentioned above imply that $N \cap H=\{1\}$. Since $|N|$ divides $7 \cdot 11 \cdot 19$, we may assume that $N$ is cyclic of prime order. Since $A_{5}$ centralizes $N$, and $G$ is perfect, we infer that $N$ lies in the center of $G$. On the other hand, by Schur-Zassenhaus theorem $G$ splits over $N$ and consquently $N$ is a direct factor of $G$. This contradicts the fact that $G$ is perfect. Hence $G$ is indeed a simple group.

(15.4) As a final example we consider the presentation (10.1) of $H S$. Let $G=\langle R: S\rangle$ be the group defined by that presentation and let

$$
H=\langle a, b, c, d, f\rangle \text { and } K=\langle a, b, c, d, e\rangle
$$

be its subgroups. By using the presentation (1.1) of $M_{11}$ we conclude that $K$ is isomorphic to $M_{11}$ or is a trivial group. A coset enumeration shows that the index $[G: K]$ is 5,600 , that is, it is equal to the index of $M_{11}$ in $H S$. Next we define a permutation representation $\phi$ of $G$ on the right cosets of $H$ by using CAYLEY. We then find that the degree of $\operatorname{Im} \phi$ is 100 and that it has the same order as $H S$. Hence we can conclude that $K \cong M_{11}$ and the $\phi$ is faithful. Furthermore, still using CAYLEY, we find that the orbits of $H$ have the sizes 
1,22 , and 77. Since these properties characterize $H S$, we conclude that indeed $G \cong H S$.

\section{Added in proof}

By omitting, in the presentation (13.2) of $\mathrm{Co}_{3}$, the generator $d$ and all the defining relators which involve that generator, we obtain a presentation of $2 \cdot S_{6}(2)$ of length 125 .

\section{References}

[1] J. J. Cannon, 'An introduction to the group theory language Cayley', Computational Group Theory, (Academic Press, 1984).

[2] J. H. Conway, 'The miracle octad generator', Topics in Group Theory and Computation, Proceedings of the Summer School, Galway, 1973, pp. 62-68, (Academic Press, 1977).

[3] J. H. Conway, 'Hexacode and tetracode -MOG and MINIMOG', Computational Group Theory, (Academic Press, 1984).

[4] J. H. Conway. R. T. Curtis, S. P. Norton, R. A. Parker and R. A. Wilson, Atlas of Finite Groups, (Clarendon Press, Oxford, 1985).

[5] R. T. Curtis, 'A new combinatorial approach to $M_{24}$ ', Math. Proc. Cambridge Philos. Soc. 79 (1976), 25-42.

[6] L. Soicher, Presentations of some finite groups, (Ph.D. Thesis, Cambridge, 1985).

[7] - 'Presentations for some groups related to $\mathrm{Co}_{1}$ ', preprint.

[8] M. F. Warboys, 'Generators for the sporadic group $\mathrm{Co}_{3}$ as a $(2,3,7)$-group', Proc. Edinburgh Math. Soc. (2) 25 (1982), 65-68.

Department of Pure Mathematics

University of Waterloo

Waterloo, Ontario

Canada N2L 3G1 